Frontiers of Information Technology \& Electronic Engineering www.jzus.zju.edu.cn; engineering.cae.cn; www.springerlink.com ISSN 2095-9184 (print); ISSN 2095-9230 (online)

E-mail: jzus@zju.edu.cn

\title{
Erratum:
}

\section{Erratum to: Past review, current progress, and challenges ahead on the cocktail party problem}

\author{
Yan-min QIAN ${ }^{\dagger * 1}$, Chao $\mathrm{WENG}^{2}$, Xuan-kai CHANG ${ }^{1}$, Shuai WANG ${ }^{1}$, Dong YU ${ }^{2}$ \\ ${ }^{1}$ Department of Computer Science and Engineering, Shanghai Jiao Tong University, Shanghai 200240, China \\ ${ }^{2}$ Tencent AI Lab, Tencent, Bellevue 98004, USA \\ †E-mail: yanminqian@sjtu.edu.cn
}

https://doi.org/10.1631/FITEE.17e0814

Erratum to: Front Inform Technol Electron Eng, 2018 19(1):40-63

https://doi.org/10.1631/FITEE.1700814

In the original version of this article, the affiliations are incorrect. The correct affiliations are given above.
The corresponding author's E-mail address should be yanminqian@sjtu.edu.cn. 